\title{
Identification of a suitable endogenous control gene in porcine blastocysts for use in quantitative PCR analysis of microRNAs
}

\author{
LI Jun ${ }^{1}$, WEI HengXi ${ }^{1,3}$, LI Yan ${ }^{2}$, LI QiuYan ${ }^{1,2} \&$ LI Ning ${ }^{1 *}$ \\ ${ }^{1}$ State Key Laboratory for Agrobiotechnology, College of Biological Science, China Agricultural University, Beijing 100193, China; \\ ${ }^{2}$ GenProtein Biotech Ltd., Beijing 100193, China; \\ ${ }^{3}$ College of Animal Science and Technology, China Agricultural University, Beijing 100193, China
}

Received September 3, 2011; accepted January 17, 2012

\begin{abstract}
To obtain reliable results in quantitative PCR (qPCR) reactions, an endogenous control (EC) gene is needed to correct for systematic variations. In this study, a TaqMan low density array was used to quantify the expression levels of microRNA (miRNA) genes in in vivo fertilized, in vitro fertilized, parthenogenetic and somatic cell nuclear transfer blastocysts. The aim was to identify suitable EC genes for the qPCR analysis of miRNAs in porcine blastocysts. The results showed that thirty-six miRNAs were commonly expressed in the four kinds of embryos and the expression levels of eleven miRNAs were similar in the different embryo types ( $P$-value $>0.05)$. These 11 miRNAs were selected as candidate EC genes for further analysis and, of these, miR-16 was identified as the most stable EC gene by the GeNorm (a tool based on a pair-wise comparison model that calculates the internal control genes stability measure and determines the most reliable pair of EC genes) and NormFinder (an excel plug-in that uses an ANOVA-based model to estimate intra- and inter- group variation to indicate the single most stable EC gene) programs. In addition, a cell number normalization method validated miR-16 as a suitable EC gene for use in future qPCR analysis of miRNAs in porcine blastocysts.
\end{abstract}

endogenous control gene, quantitative PCR, microRNA, blastocyst

Citation: Li J, Wei H X, Li Y, et al. Identification of a suitable endogenous control gene in porcine blastocysts for use in quantitative PCR analysis of microRNAs. Sci China Life Sci, 2012, 55: 126-131, doi: 10.1007/s11427-012-4289-8

MicroRNAs (miRNAs) are a large family of short noncoding RNAs ( 22 nucleotides), and in mammals, more than 400 different miRNAs have been identified, most of which are well conserved in the different species [1]. MiRNAs are initially transcribed as long primary transcripts (pri-miRNAs) that are processed first by the RNase enzyme complex, Drosha-DGCR8, and then by Dicer, leading to the incorporation of a single strand into the RNA-induced silencing complex [2]. By pairing with partially complementary sequences in the $3^{\prime}$-untranslated regions of the target mRNAs, miRNAs can either repress translational efficiency or induce transcript decay [3]. It is estimated that at least

*Corresponding author (email: ninglcau@cau.edu.cn)
$20 \%$ of human genes are targeted by miRNAs [4]. MiRNAs have been implicated in regulating embryonic stem cell differentiation [5-7], DNA methylation [8-10], and early embryonic development [11,12].

Real-time quantitative PCR (qPCR) is by far the preferred method for miRNA expression quantification studies because of its sensitivity, specificity and wide dynamic range [13]. In qPCR experiment, several variables including amount and quality of RNA, enzymatic efficiencies and differences between tissues or cells in overall transcriptional activity need to be normalized to produce reliable data and incorporating an endogenous control (EC) gene has been found to be an effective method [14]. Because miRNAs are expressed in a tissue-specific pattern [15], there is currently 
no consensus on suitable EC genes for the quantitative analysis of miRNAs. To identify suitable EC genes for the qPCR analysis of miRNAs in different kinds of porcine blastocysts, the present study was conducted to quantify the expression levels of the miRNAs in in vivo fertilized (IVO), in vitro fertilized (IVF), parthenogenetic (PA) and somatic cell nuclear transfer (SCNT) blastocysts.

\section{Materials and methods}

\subsection{Generation of porcine IVO, IVF, PA and SCNT blastocysts}

Porcine IVO, IVF, PA and SCNT blastocysts were used for the miRNA profiling assays. Ten embryos were chosen per group in two replicates for the TaqMan Array experiment.

Animals were treated according to the China Agricultural University animal care protocol and guidelines. All chemicals were purchased from Sigma (St. Louis, MO, USA), unless otherwise stated.

Black-coated Xiang pigs were bred in the China Agricultural University and used to collect the in vivo blastocysts. Estrous sows were mated with breeding boar twice with a $12 \mathrm{~h}$ interval. On day 7 after the mating, the sows $(n=20)$ were electrically stunned and exsanguinated. The genital tracts were removed immediately after death, and the IVO blastocysts were recovered by flushing the uterine horn with Dulbecco's phosphate buffered saline (DPBS) supplemented with $0.1 \%$ BSA.

SCNT and PA embryos were produced based on a previous method [16]. In brief, in vitro matured oocytes were treated with $10 \mu \mathrm{g} \mathrm{mL}^{-1}$ Hoechst33342 for 5-10 min, and enucleated under a fluorescence microscope equipped with a manipulation system (Eppendorf, Germany). Then, a single fibroblast cell was injected into the perivitelline space of the enucleated oocyte. After $1 \mathrm{~h}$ recovery in HEPES buffered NCSU-23, the reconstructed couplets were fused in a fusion medium $\left(0.25 \mathrm{~mol} \mathrm{~L}{ }^{-1}\right.$ mannitol, $0.1 \mathrm{mmol} \mathrm{L}^{-1} \mathrm{CaCl}_{2}$, $0.1 \mathrm{mmol} \mathrm{L}^{-1} \mathrm{MgCl}_{2}, 0.5 \mathrm{mmol} \mathrm{L}{ }^{-1}$ HEPES and $0.1 \mathrm{mg}$ $\mathrm{mL}^{-1}$ PVA) with two DC pulses of $1.6 \mathrm{kV} \mathrm{cm}^{-1}$ for $100 \mu \mathrm{s}$ each with a $100 \mu$ s interval. The fused embryos were transferred into porcine zygote medium 3 (PZM3) supplemented with $10 \mu \mathrm{g} \mathrm{mL}^{-1}$ cytochalasin $\mathrm{B}(\mathrm{CB})$ and $10 \mu \mathrm{g} \mathrm{mL}^{-1}$ cycloheximide (CHX) and cultured at $39^{\circ} \mathrm{C}$ in $5 \% \mathrm{CO}_{2}, 5 \% \mathrm{O}_{2}$ and $90 \% \mathrm{~N}_{2}$ with $100 \%$ humidity for $4 \mathrm{~h}$, and then again transferred and cultured in PZM3. Parthenogenetic activation was conducted using the same activation parameters as were used for the SCNT blastocysts. The IVF embryo production was conducted based on our previous report [17]. Briefly, oocytes matured in vitro for $42-44 \mathrm{~h}$ were digested and pipetted in $0.1 \%$ hyaluronidase to remove the cumulus cells. After washings, the oocytes were placed in drops of IVF medium which had been kept for $18-24 \mathrm{~h}$ in the incubator before use. Then the fresh spermatozoa were washed, centrifuged and resuspended in the IVF medium, before being added to the drops of IVF medium containing the oocytes with a final concentration of spermatozoa of $1-1.5 \times 10^{6} \mathrm{~mL}^{-1}$. After co-incubation for $6 \mathrm{~h}$, the putative zygotes were washed three times and transferred to the PZM3 for further cultures. Blastocysts of the IVO, SCNT, PA and IVF embryos with good morphology were collected in DEPC-PBS and stored at $-80^{\circ} \mathrm{C}$.

\subsection{Low density miRNA TaqMan Array}

The low density miRNA TaqMan Array functions as an array of reaction vessels for the PCR step based on the multiplex stem-loop qRT-PCR format $[18,19]$. In the low density miRNA TaqMan Array experiment, 10 blastocysts per embryo group were used and the experiment was performed twice. Because of the small number of cells used in the experiment, all the miRNAs were released from the blastocysts using the cell heat lysate method [18]; the obtained samples were directly used as templates for reverse transcription (RT). The cycle parameters for the RT reaction were $16^{\circ} \mathrm{C}$ for $30 \mathrm{~min}, 42^{\circ} \mathrm{C}$ for $30 \mathrm{~min}, 85^{\circ} \mathrm{C}$ for $5 \mathrm{~min}$, and hold at $4^{\circ} \mathrm{C}$. The miRNA expression profile was obtained using the TaqMan Array Human MicroRNA Panel v1.0 containing 365 miRNA targets (Applied Biosystems, Foster City, CA). Gene expression levels were quantified using the ABI Prism 7900HT sequence detection system. Cycling conditions were as follows: $95^{\circ} \mathrm{C}$ for $10 \mathrm{~min}$, followed by 40 cycles of $95^{\circ} \mathrm{C}$ for $15 \mathrm{~s}$ and $60^{\circ} \mathrm{C}$ for $60 \mathrm{~s}$. The array experiments were performed twice using samples from independent biological experiments.

\subsection{Method for endogenous control (EC) gene selection}

Threshold cycle $\left(C_{\mathrm{t}}\right)$ values generated by qPCR reaction were converted to relative quantities using the formula $2^{-\Delta C_{\mathrm{t}}}$ recommended by Livak et al. [20] with $\Delta C_{\mathrm{t}}=$ average $C_{\mathrm{t}}$ test sample-average $\mathrm{Ct}$ calibrator sample.

\subsection{GeNorm and NormFinder programs}

Stability of EC expression was analyzed using two programs, GeNorm and NormFinder. The GeNorm tool which is based on a pair-wise comparison model was used to calculate the internal control genes-stability measure (M) to determine the most reliable pair of EC genes [21]. NormFinder, an excel plug-in in the ANOVA-based model, was used to estimate intra- and inter- group variation. NormFinder ranks candidate control genes by estimating their expression stability and indicates the single most stable EC gene [22].

\subsection{Q-PCR data analysis}

Expression levels of the miRNAs were calculated utilizing 
the $2^{-\Delta \Delta C_{\mathrm{t}}}$ method, with $\Delta \Delta C_{\mathrm{t}}=\left(C_{\mathrm{t}}\right.$ target gene, test sample- $C_{\mathrm{t}}$ endogenous control gene, test sample $)-\left(C_{\mathrm{t}}\right.$ target gene, calibrator sample- $C_{\mathrm{t}}$ endogenous control gene, calibrator sample). In the present study, the IVO blastocysts were used as the calibrator sample.

\subsection{Q-PCR measurement for single miRNA}

A single blastocyst from each embryo type was collected as mentioned above. After cell lysis all the miRNAs were released and used as templates for the $\mathrm{RT}$ reaction. The cycle parameters for the RT and PCR reactions were the same as those used in the TaqMan Array experiment. All reactions were run in triplicate. The $2^{-\Delta \Delta C_{\mathrm{t}}}$ formula was used to calculate the relative quantities. Lin- 4 miRNA was diluted in concentration gradients and used to draw the standard curves.

\subsection{Statistical analysis}

One-way ANOVA (SPSS 16.0, SPSS Inc. Chicago, USA) was used to determine differences in the relative expression levels of the miRNAs between different groups of blastocysts. A $P$-value $<0.05$ was considered to be statistically significant.

\section{Results}

\subsection{Developmental potential of the different embryo types}

Blastocyst formation rate and cell number in the different embryo types are summarized in Table 1. As shown, the percentage of blastocyst in the SCNT embryos was significantly lower than the percentage in the IVF and PA embryos $(P<0.05)$ and the number of blastocysts in the SCNT embryos was also significantly lower than in the other two types of embryos $(P<0.05)$.

\subsection{Eleven candidate EC genes that were not differen- tially expressed in the blastocysts}

Thirty-six miRNAs were found to be commonly expressed in the four kinds of blastocysts (Table 2). The relative quan-

Table 1 Development rate and blastocyst cell number in the different embryo types ${ }^{\text {a) }}$

\begin{tabular}{cccc}
\hline Embryo type & Total & $\begin{array}{c}\text { No. blastocysts } \\
(\%)\end{array}$ & $\begin{array}{c}\text { No. cells in blastocysts } \\
(\text { No. embryos tested) }\end{array}$ \\
\hline SCNT & 498 & $52(10.44)^{\mathrm{a}}$ & $36.38 \pm 4.33^{\mathrm{a}}(13)$ \\
PA & 205 & $66(32.20)^{\mathrm{b}}$ & $43.50 \pm 4.20^{\mathrm{b}}(16)$ \\
IVF & 398 & $65(16.33)^{\mathrm{c}}$ & $47.12 \pm 4.95^{\mathrm{c}}(17)$ \\
\hline
\end{tabular}

a) Values with different superscripts in the same column are significantly different $(P<0.05)$.
Table 2 MiRNAs that were commonly expressed in the four kinds of blastocysts

\begin{tabular}{cc}
\hline Group (number) & miRNAs \\
\hline $\begin{array}{c}\text { Commonly expressed miRNAs in the } \\
\text { four kinds of blastocysts (36) }\end{array}$ & miR-103, miR-106b, miR-125a, \\
miR-130a, miR-130b, miR-133b, \\
miR-148a, miR-151, miR-15b, \\
miR-16, miR-182, miR-186, \\
miR-191, miR-19a, miR-19b, \\
miR-203, miR-20a, miR-222, \\
miR-24, miR-25, miR-26a, miR-26b, \\
miR-28, miR-301, miR-302b, \\
miR-302c, miR-30a-5p, miR-30b, \\
miR-30c, miR-30d, miR-367, \\
miR-374, miR-378, miR-484, \\
miR-92, miR-93
\end{tabular}

tities of these 36 miRNAs were calculated using the $2^{-\Delta C_{t}}$ method; the IVO blastocysts were used as the calibrator sample (Figure 1). Eleven miRNAs (miR-148a, miR-16, miR-186, miR-20a, miR-24, miR-26a, miR-26b, miR-302b, miR-302c, miR-374 and miR-92) were not differentially expressed in the four kinds of embryos $(P>0.05)$ (Figure 1). Because EC genes should be stably expressed in the test samples, we selected these 11 miRNAs as candidate EC genes for further analysis.

\subsection{MiR-16 as the most suitable EC gene for qPCR in porcine embryos}

The GeNorm analysis showed that miR-374 was the least stable of the 11 miRNA genes, while miR-16 and miR-302b were the two genes that were most stably expressed (Figure 2). Because the GeNorm program requires a pair of control genes to calculate $M$, the two most stably expressed EC genes could not be ranked any further. For further analysis, the NormFinder program was used. A stability value of 0.022 was obtained for the miR-16 gene, identifying it as the most stably expressed of the 11 candidate EC genes (the lower the stability value, the higher the gene stability). The results of the ranking of the candidate EC genes using these two programs are shown in Table 3 . The miR-16 gene was therefore selected as the EC gene to be used in our research.

\subsection{Validation of the GeNorm and NormFinder results using a cell number normalization method}

One of the most important roles of the EC gene is to correct for variations caused by different cell numbers; in this study, the variations are in the number of cells in the four kinds of blastocysts. Therefore, we proposed that the results of EC normalization should be consistent with the results normalized by cell number. To validate the results obtained using the GeNorm and NormFinder programs, we determined the expression levels of the miR-27a gene in the blastocysts by both the cell number normalization method and the EC method. As shown in Figure 3, miR-27a was found by both methods to be significantly expressed in the SCNT blastocysts compared with in the other embryo types $(P<0.01)$. 


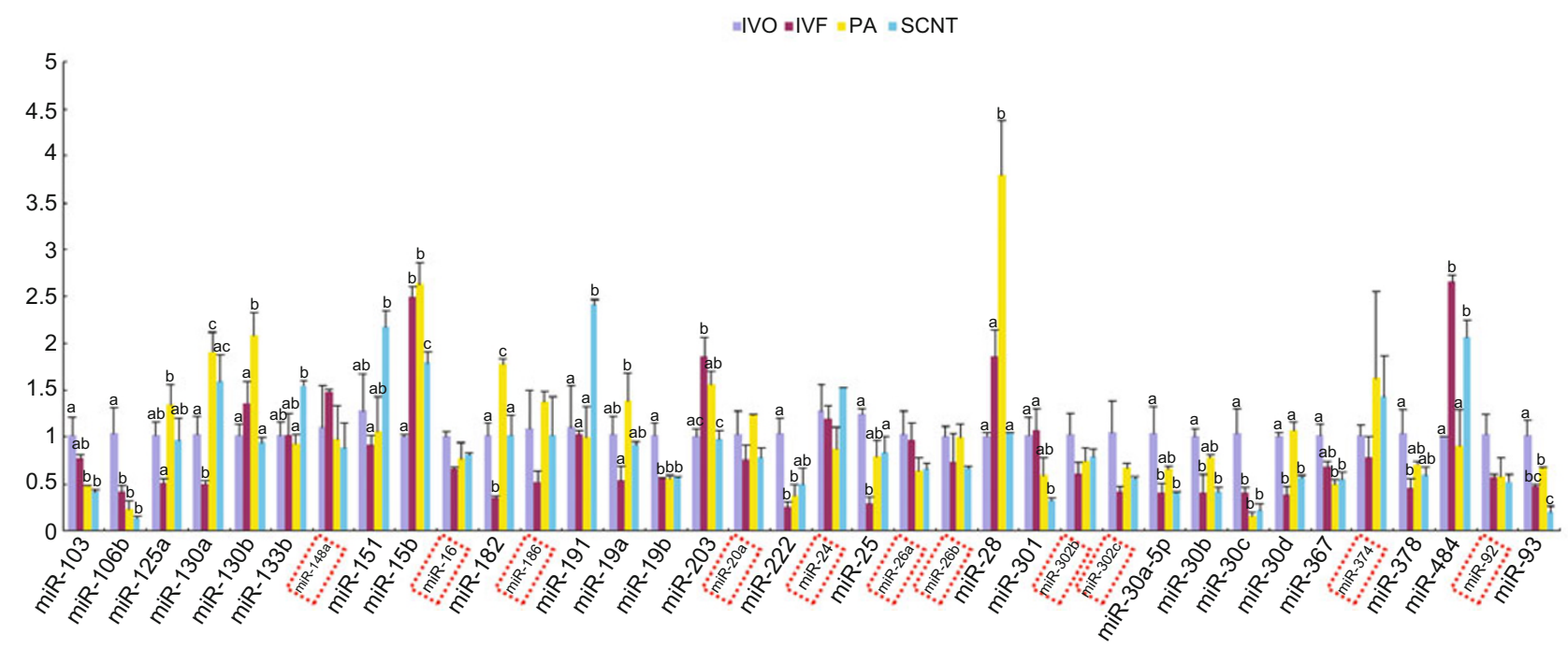

Figure 1 Relative quantification of the 36 miRNAs that were commonly expressed in the four kinds of blastocysts. The red box indicates the 11 miRNAs that were not differentially expressed in the four different blastocyst types.
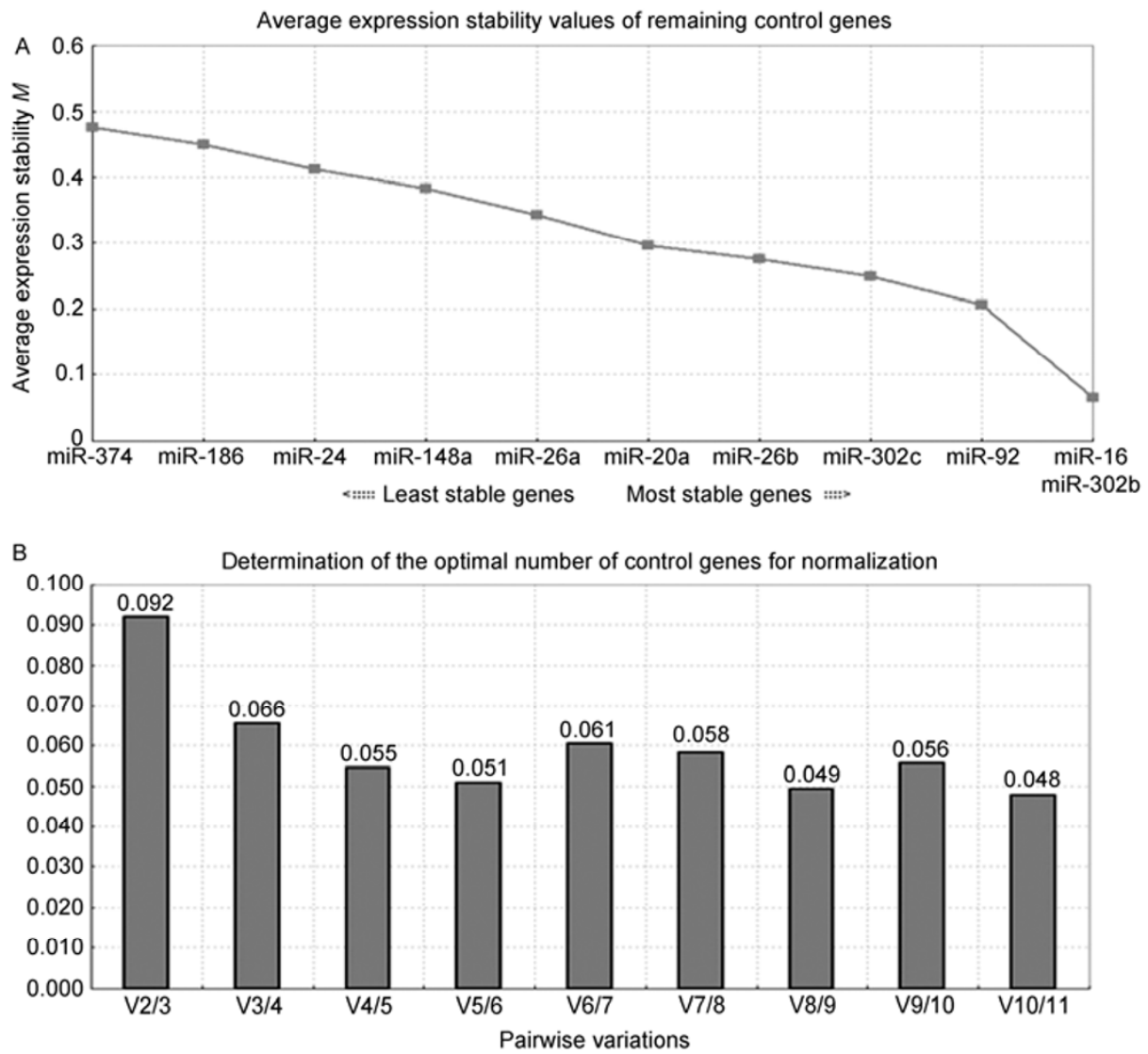

Figure 2 GeNorm analysis of the stability of the candidate EC genes. A, Average expression stability of the EC candidates calculated using GeNorm. A stability value $(M)$ was calculated for each candidate EC. The least stable gene with the highest $M$ value was automatically excluded and $M$ values was recalculated for the remaining EC genes, ultimately resulting in a stability value for the two most stable EC genes (miR-16 and miR-302b). B, Determination of the optimal number of EC genes for normalization. The $V$ value defines the pairwise variation between two sequential normalization factors. The least number of genes for each $V<0.15$ was selected as the optimal set of genes for normalization. 
Table 3 Ranking of candidate EC genes using the NormFinder and GeNorm programs

\begin{tabular}{cccccc}
\hline \multirow{2}{*}{ Rank } & \multicolumn{2}{c}{ NormFinder } & & \multicolumn{2}{c}{ GeNorm } \\
\cline { 2 - 3 } \cline { 5 - 6 } & $\begin{array}{c}\text { Candidate EC } \\
\text { genes }\end{array}$ & Stability & & $\begin{array}{c}\text { Candidate EC } \\
\text { genes }\end{array}$ & Stability $(M)$ \\
\hline 1 & miR-16 & 0.022 & & miR-16 & 0.350 \\
2 & miR-302b & 0.065 & & miR-302b & 0.363 \\
3 & miR-26b & 0.113 & & miR-26b & 0.387 \\
4 & miR-20a & 0.166 & & miR-20a & 0.420 \\
5 & miR-92 & 0.206 & & miR-92 & 0.441 \\
6 & miR-302c & 0.247 & & miR-302c & 0.479 \\
7 & miR-26a & 0.291 & & miR-26a & 0.514 \\
8 & miR-24 & 0.305 & & miR-24 & 0.549 \\
9 & miR-148a & 0.336 & & miR-148a & 0.568 \\
10 & miR-186 & 0.344 & & miR-186 & 0.572 \\
11 & miR-374 & 0.356 & & miR-374 & 0.594 \\
\hline
\end{tabular}

\section{Discussion}

Ribosomal RNAs (rRNA), small nuclear RNA (snRNA), small nucleolar RNAs (snoRNAs) and U6 snRNA (RNU6B) have been commonly used as EC genes in the qPCR analysis of miRNAs. However, concerns have been expressed regarding the use of rRNAs in normalization strategies because they are often expressed at much higher levels than the target miRNAs making it very difficult to quantify an rRNA and a rare miRNA transcript in the same RNA dilution [23]. Further, it has been reported that snRNAs and snoRNAs may exhibit tissue-specific and developmental regulation [24] and RNU6B was found to be less stably expressed than the other genes [25]. Thus the genes that have been used as EC genes are no longer thought to be suitable for the quantification of miRNAs making it was necessary to establish the most suitable EC genes in individual experiments. In this study, miR-16 was identified as the most suitable EC gene in porcine blastocysts and to our knowledge this is the first report of the establishment of reliable EC genes for the qPCR analysis of miRNAs in porcine embryos.

GeNorm and NormFinder software have been widely used to identify suitable EC genes for qPCR experiments. GeNorm generates a gene-stability measure $(M)$ which can be defined as the average pairwise variation $(V)$ for one candidate EC gene compared to all other candidate EC genes. Stepwise exclusion of the gene with the highest $M$ value results in the recalculation of $M$ values till the most stable pair remains [21]. In GeNorm, a normalization factor $(\mathrm{NF})$ is generated for each sample using the geometric average of the expression of the most stable EC genes. The pairwise variation value, $V$ is the variation between two sequential NFs $(V n / n+1$, where $n=$ the number of ECs used). The least number of genes for each $V<0.15$ was selected as the optimal set of genes for normalization. Although it has been reported that the more EC genes that are used in the calculation, the more reliable the result, considering the cost and complexity in conducting the experiment and data analysis, fewer EC genes might be recommended. In our experiment, because the $V$ value of two candidate EC genes was 0.092 , much lower than 0.15 , it was proposed that, in our study, one EC gene should be adequate to correct for any variations between the embryo types. Because the GeNorm program requires a pair of control gene to calculate $M$, the two most stably expressed EC genes cannot be ranked any further. Therefore, to identify the most stably expressed EC gene, NormFinder was used to rank the same 11 candidate EC genes that were ranked by GeNorm. NormFinder ranked miR-16 as the most stable of the candidate EC genes.

In the present study, the aim was to identify an EC gene that could be used to correct for variations caused by different cell numbers in the four kinds of blastocysts to be studied. For the EC gene to be considered suitable, the results normalized by the selected EC gene had to be consistent with the results corrected by the blastocyst cell numbers. To validate the selection of miR-16 as the EC gene, the expression levels of miR-27a in the four kinds of blastocysts were analyzed using both normalization methods. As shown in Figure 3, the two methods produced similar results, firmly establishing miR-16 as a suitable EC gene for the qPCR analysis of miRNAs in porcine blastocysts.
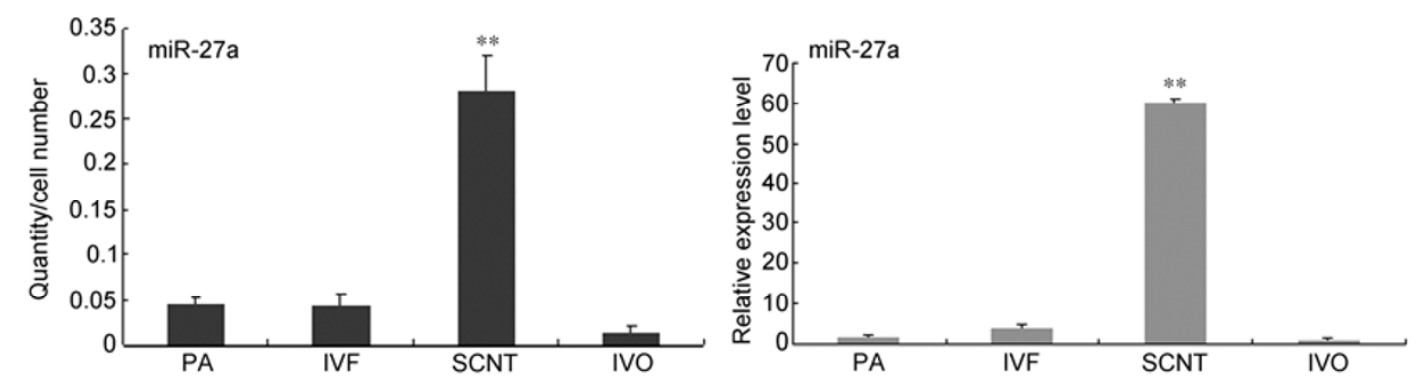

Figure 3 MiR-27a expression levels in the four kinds of blastocysts using two different normalization methods. A, The miR-27a expression levels were normalized using blastocyst cell number. B, The miR-27a expression levels were normalized using miR-16 as the EC gene. 
This work was supported by the National Basic Research Program of China (Grant Nos. 2006CB102106 and 2007CB947402) and the Foundation for Innovative Research Groups of the National Natural Science Foundation of China (Grant No. 30621064).

1 Brown B D, Naldini L. Exploiting and antagonizing microRNA regulation for therapeutic and experimental applications. Nat Rev Genet, 2009, 10: 578-585

2 Cordes K R, Sheehy N T, White M P, et al. miR-145 and miR-143 regulate smooth muscle cell fate and plasticity. Nature, 2009, 460: 705-710

3 Eulalio A, Huntzinger E, Izaurralde E. Getting to the root of miRNA-mediated gene silencing. Cell, 2008, 132: 9-14

4 Xie X, Lu J, Kulbokas E J, et al. Systematic discovery of regulatory motifs in human promoters and $3^{\prime}$ UTRs by comparison of several mammals. Nature, 2005, 434: 338-345

5 Foshay K M, Gallicano G I. miR-17 family miRNAs are expressed during early mammalian development and regulate stem cell differentiation. Dev Biol, 2009, 326: 431-443

6 Kanellopoulou C, Muljo S A, Kung A L, et al. Dicer-deficient mouse embryonic stem cells are defective in differentiation and centromeric silencing. Genes Dev, 2005, 19: 489-501

7 Murchison E P, Partridge J F, Tam O H, et al. Characterization of Dicer-deficient murine embryonic stem cells. Proc Natl Acad Sci USA, 2005, 102: 12135-12140

8 Benetti R, Gonzalo S, Jaco I, et al. A mammalian microRNA cluster controls DNA methylation and telomere recombination via Rbl2dependent regulation of DNA methyltransferases. Nat Struct Mol Biol, 2008, 15: 268-279

9 Duursma A M, Kedde M, Schrier M, et al. miR-148 targets human DNMT3b protein coding region. RNA, 2008, 14: 872-877

10 Fabbri M, Garzon R, Cimmino A, et al. MicroRNA-29 family reverts aberrant methylation in lung cancer by targeting DNA methyltransferases 3A and 3B. Proc Natl Acad Sci USA, 2007, 104: 15805-15810

11 Bernstein E, Kim S Y, Carmell M A, et al. Dicer is essential for mouse development. Nat Genet, 2003, 35: 215-217

12 Tang F, Kaneda M, O'Carroll D, et al. Maternal microRNAs are essential for mouse zygotic development. Genes Dev, 2007, 21: 644-648

13 Davoren P A, McNeill R E, Lowery A J, et al. Identification of suitable endogenous control genes for microRNA gene expression analysis in human breast cancer. BMC Mol Biol, 2008, 9: 76

14 Stamova B S, Apperson M, Walker W L, et al. Identification and validation of suitable endogenous reference genes for gene expression studies in human peripheral blood. BMC Med Genomics, 2009, 2: 49

15 Reddy A M, Zheng Y, Jagadeeswaran G, et al. Cloning, characterization and expression analysis of porcine microRNAs. BMC Genomics, 2009, 10: 65

16 Wei H X, Zhang K, Ma Y F, et al. Stage-dependent effect of leptin on development of porcine embryos derived from parthenogenetic activation and transgenic somatic cell nuclear transfer. J Reprod Dev, 2009, 55: 99-104

17 Zhang K, Wei H X, Zhang Y H, et al. Effects of ghrelin on in vitro development of porcine in vitro fertilized and parthenogenetic embryos. J Reprod Dev, 2007, 53: 647-653

18 Chen C, Ridzon D A, Broomer A J, et al. Real-time quantification of microRNAs by stem-loop RT-PCR. Nucleic Acids Res, 2005, 33: e179

19 Tang F, Hajkova P, Barton S C, et al. MicroRNA expression profiling of single whole embryonic stem cells. Nucleic Acids Res, 2006, 34: e9

20 Livak K J, Schmittgen T D. Analysis of relative gene expression data using real-time quantitative PCR and the 2(-Delta Delta $\mathrm{C}(\mathrm{T})$ ) Method. Methods, 2001, 25: 402-408

21 Vandesompele J, De Preter K, Pattyn F, et al. Accurate normalization of real-time quantitative RT-PCR data by geometric averaging of multiple internal control genes. Genome Biol, 2002, 3: research0034research0034.11

22 Andersen C L, Jensen J L, Orntoft T F. Normalization of real-time quantitative reverse transcription-PCR data: a model-based variance estimation approach to identify genes suited for normalization, applied to bladder and colon cancer data sets. Cancer Res, 2004, 64: 5245-5250

23 Wong M L, Medrano J F. Real-time PCR for mRNA quantitation. Biotechniques, 2005, 39: 75-85

24 Runte M, Huttenhofer A, Gross S, et al. The IC-SNURF-SNRPN transcript serves as a host for multiple small nucleolar RNA species and as an antisense RNA for UBE3A. Hum Mol Genet, 2001, 10: 2687-2700

25 Peltier H J, Latham G J. Normalization of microRNA expression levels in quantitative RT-PCR assays: identification of suitable reference RNA targets in normal and cancerous human solid tissues. RNA, 2008, 14: 844-852

Open Access This article is distributed under the terms of the Creative Commons Attribution License which permits any use, distribution, and reproduction in any medium, provided the original author(s) and source are credited. 